\title{
Kinematics of Tycho-2 Red Giant Clump Stars
}

\author{
V.V. Bobylev, A.S. Stepanishchev, A.T. Bajkova, and G.A. Gontcharov \\ Pulkovo Astronomical Observatory, Russian Academy of Sciences, St-Petersburg
}

\begin{abstract}
Based on the Ogorodnikov-Milne model, we analyze the proper motions of 95 633 red giant clump(RGC) stars from the Tycho-2 Catalogue. The following Oort constants have been found: $A=15.9 \pm 0.2 \mathrm{~km} \mathrm{~s}^{-1} \mathrm{kpc}^{-1}$ and $B=-12.0 \pm 0.2 \mathrm{~km} \mathrm{~s}^{-1} \mathrm{kpc}^{-1}$. Using 3632 RGC stars with known proper motions, radial velocities, and photometric distances, we show that, apart from the star centroid velocity components relative to the Sun, only the model parameters that describe the stellar motions in the $X Y$ plane differ significantly from zero. We have studied the contraction (a negative $K$ effect) of the system of RGC stars as a function of their heliocentric distance and elevation above the Galactic plane. For a sample of distant (500-1000 pc) RGC stars located near the Galactic plane $(|Z|<200$ pc) with an average distance of $d=0.7 \mathrm{kpc}$, the contraction velocity is shown to be $K d=-3.5 \pm 0.9$ $\mathrm{km} \mathrm{s}^{-1}$; a noticeable vertex deviation, $l_{x y}=9^{\circ} \pm 0.5^{\circ}$, is also observed for them. For stars located well above the Galactic plane $(|Z| \geq 200 \mathrm{pc})$, these effects are less pronounced, $K d=-1.7 \pm 0.5 \mathrm{~km} \mathrm{~s}^{-1}$ and $l_{x y}=4^{\circ} .9 \pm 0^{\circ} .6$. Using RGC stars, we have found a rotation around the Galactic $X$ axis directed toward the Galactic center with an angular velocity of $-2.5 \pm 0.3 \mathrm{~km} \mathrm{~s}^{-1} \mathrm{kpc}^{-1}$, which we associate with the warp of the Galactic stellar-gaseous disk.
\end{abstract}

\section{INTRODUCTION}

The red giant clump (RGC) stars are distributed fairly uniformly over the celestial sphere. Since they occupy a compact region on the Hertzsprung-Russell diagram and, hence, have the same luminosity, reliable photometric distance estimates can be obtained for them. These stars are of great interest in analyzing the three-dimensional kinematics of various Galactic subsystems.

First of all, we are interested in such peculiarities as the expansion/contraction of stellar groups, the deviation of their vertex from the direction of the kinematic center, i.e., the Galactic center, and the connection of the stellar kinematics with the warp of the Galactic stellar-gaseous disk.

Various authors have found a negative value of the Oort parameter $\mathrm{K}$ responsible for the expansion/contacrtion. This means that the system of stars is in the state of contraction. We are talking about the plane effect that describes the contraction in the Galactic $X Y$ plane. The negative $\mathrm{K}$ effect in the motion of distant OB stars was detected by Torra et al. (2000) and Bobylev (2004). It was also revealed in the motion of open star clusters (Bobylev et al. 2007) and in the motion of dwarf stars (Rybka 2004a) and giant stars (Rybka 2004b, 2008). 
At present, the nature of the negative $\mathrm{K}$ effect is not completely clear. It is hypothesized that this effect is related to: (i) the peculiarities of stellar radial velocity measurements (Pont et al. 1994), (ii) the influence of the bar at the Galactic center (Point et al. 1994), and (iii) the influence of the Galactic spiral structure (Rohlfs 1977; Metzger et al. 1998). In particular, Fernández et al. (2001) and Bobylev et al. (2006a) made an attempt to take into account the influence of a spiral density wave; it failed, because the negative $\mathrm{K}$ effect was retained.

Rybka (2008) showed that the RGC stars have different vertex deviations and differ significantly by the magnitude of the $\mathrm{K}$ effect, depending on the Galactic latitude. In this respect, it is interesting to trace the changes in kinematic parameters of these stars located in various layers parallel to the Galactic plane.

Analysis of the large-scale structure of neutral hydrogen has shown that the gaseous disk in the Galaxy is warped (for a review, see Burton, 1988). The results of studying this structure on the basis of currently available data on the HI distribution are presented in Kalberla and Dedes (2008). This structure is revealed by the spatial distribution of stars and dust (Drimmel and Spergel 2001) and by the distribution of pulsars in the Galaxy (Yusifov 2004). Having analyzed nearby Hippacros (1997) stars, Dehnen (1998) showed that the distribution of their residual velocities $V_{y}-V_{z}$ was in satisfactory agreement with various rotation models of the warped disk. Miyamoto et al. (1993) and Miyamoto and Zhu (1998) determined the rotation parameters of the warped stellar-gaseous disk by analyzing giant stars of various spectral types and samples of Hipparcos O-B5 stars. Thus, there is positive experience in solving this problem using data on stars relatively close to the Sun.

The goal of this paper is to study the kinematic peculiarities of a wide solar neighborhood on the basis of data on the RGC stars from the Tycho-2 Catalogue (Hog et al. 2000) selected by Gontcharov (2008) and, in particular, to establish the connection of the kinematics of RGC stars with the warp of the Galactic stellar-gaseous disk.

\section{DATA}

At present, two lists of candidates for RGC stars compiled from a combination of Tycho-2 and 2MASS data (Skrutskie et al. 2006) have been published. These are the catalogs by Rybka (2006, 2007) and Gontcharov (2008).

The catalog by Rybka (2007) contains about 60000 RGC candidates from Tycho-2 with photometric characteristics from 2MASS with distances $>100 \mathrm{pc}$. The catalog by Gontcharov (2008) contains about 97000 stars with data from the same catalogs as those of Rybka but without any distance constraint. Therefore, the number of stars is larger. In contrast to the catalog by Rybka (2007), the catalog by Gontcharov (2008) gives a photometric distance estimate for each of the stars corrected for interstellar extinction. In this respect, the catalog by Gontcharov (2008) is more convenient for solving the kinematic problems considered here.

The radial velocities taken from the PCRV (Gontcharov 2006; Bobylev et al. 2006b) and RAVE (Steinmetz et al. 2006) catalogs are given for 4163 stars. These are relatively close stars with known Hipparcos trigonometric parallaxes.

Figure 1 gives an idea of the three-dimensional spatial distribution of stars. The left, middle, and right columns present the distributions of stars in the $X Y, X Z$, and $Y Z$ planes, respectively. The rows from top to bottom provide the distributions for the complete sample 
of stars and the samples of stars with distances $d<400 \mathrm{pc}, 400<d<600 \mathrm{pc}$, and $600<d<1600 \mathrm{pc}$. The distance ranges were chosen in such a way that each sample contained approximately the same number of stars. The figures reveal some structural peculiarities of the samples. Thus, for example, clumps inclined by $\approx 20^{\circ}$ to the $X$ axis, typical of young stars associated with the Gould Belt, are clearly traceable in the $Z X$ plane (c) and (d).

\section{THE METHODS OF ANALYSIS}

In this paper, we use a rectangular Galactic coordinate system with the axes directed away from the observer toward the Galactic center $\left(l=0^{\circ}, b=0^{\circ}\right.$, the $X$ axis or axis 1$)$, along the Galactic rotation $\left(l=90^{\circ}, b=0^{\circ}\right.$, the $Y$ axis or axis 2$)$, and toward the North Galactic Pole $\left(b=90^{\circ}\right.$, the $Z$ axis or axis 3$)$.

\subsection{The Ogorodnikov-Milne Model}

Using the three-dimensional velocity field. In the linear Ogorodnikov-Milne model, we adhere to the notation that was used by Clube $(1972,1973)$. The observed velocity $\mathbf{V}(r)$ of a star with a heliocentric radius vector $\mathbf{r}$ is described, to the terms of the first order of smallness $r / R_{0} \ll 1$, by the equation in vector form

$$
\mathbf{V}(r)=\mathbf{V}_{\odot}+M \mathbf{r}+\mathbf{V}^{\prime}
$$

where $\mathbf{V}_{\odot}\left(X_{\odot}, Y_{\odot}, Z_{\odot}\right)$ is the velocity of the Sun relative to the centroid of the stars under consideration and $\mathbf{V}^{\prime}$ is the residual velocity of the star. Here, the residual stellar velocities are assumed to have a random distribution. $M$ is the displacement matrix whose components are the partial derivatives of the velocity $\mathbf{u}\left(u_{1}, u_{2}, u_{3}\right)$ with respect to the distance $\mathbf{r}\left(r_{1}, r_{2}, r_{3}\right)$, where $\mathbf{u}=\mathbf{V}(R)-\mathbf{V}\left(R_{0}\right)$, while $R$ and $R_{0}$ are the Galactocentric distances of the star and the Sun, respectively. Then,

$$
M_{p q}=\left(\frac{\partial u_{p}}{\partial r_{q}}\right)_{\circ}, \quad(p, q=1,2,3) .
$$

All nine components of the displacement matrix can be determined if the distances, radial velocities, and proper motions of the sample stars are known. In this case, the conditional equations are

$$
\begin{gathered}
V_{r}=-X_{\odot} \cos b \cos l- \\
-Y_{\odot} \cos b \sin l-Z_{\odot} \sin b+ \\
+r\left(\cos ^{2} b \cos ^{2} l M_{11}+\cos ^{2} b \cos l \sin l M_{12}+\right. \\
+\cos b \sin b \cos l M_{13}+\cos ^{2} b \sin l \cos l M_{21}+ \\
+\cos ^{2} b \sin ^{2} l M_{22}+\cos b \sin b \sin l M_{23}+ \\
+\sin b \cos b \cos l M_{31}+\cos b \sin b \sin l M_{32}+ \\
\left.+\sin ^{2} b M_{33}\right), \\
4.74 r \mu_{l} \cos b=X_{\odot} \sin l-Y_{\odot} \cos l+ \\
+r\left(-\cos b \cos l \sin l M_{11}-\cos b \sin ^{2} l M_{12}-\right.
\end{gathered}
$$




$$
\begin{gathered}
-\sin b \sin l M_{13}+\cos b \cos ^{2} l M_{21}+ \\
+\cos b \sin l \cos l M_{22}+ \\
\left.+\sin b \cos l M_{23}\right) \\
4.74 r \mu_{b}=X_{\odot} \cos l \sin b+ \\
+Y_{\odot} \sin l \sin b-Z_{\odot} \cos b+ \\
+r\left(-\sin b \cos b \cos ^{2} l M_{11}-\right. \\
-\sin b \cos b \sin l \cos l M_{12}- \\
-\sin ^{2} b \cos l M_{13}-\sin b \cos b \sin l \cos l M_{21}- \\
-\sin b \cos b \sin ^{2} l M_{22}-\sin ^{2} b \sin l M_{23}+ \\
+\cos ^{2} b \cos l M_{31}+\cos ^{2} b \sin l M_{32}+ \\
\left.+\sin b \cos b M_{33}\right)
\end{gathered}
$$

where the stellar proper motion components are in mas $\mathrm{yr}^{-1}$ (milliseconds per year), the radial velocity $V_{r}$ is in $\mathrm{km} \mathrm{s}^{-1}$, and the heliocentric distance to the star $r$ is in kpc; we either calculate the latter based on the known trigonometric parallax $r=1 / \pi$ or take the photometric distance $d_{\text {phot }}$. Equations (3)-(5) contain twelve sought-for unknowns - three components of the velocity $\mathbf{V}_{\odot}\left(X_{\odot}, Y_{\odot}, Z_{\odot}\right)$ in $\mathrm{km} \mathrm{s}^{-1}$ and nine components of $M_{p q}$ in km $\mathrm{s}^{-1} \mathrm{kpc}^{-1}$. The system of equations (3)-(5) is solved by the least squares method.

The Oort constants can be found as follows: $A=0.5\left(M_{12}+M_{21}\right), B=0.5\left(M_{21}-M_{12}\right)$, $C=0.5\left(M_{11}-M_{22}\right)$ and $K=0.5\left(M_{11}+M_{22}\right)$; we determine the vertex deviation $l_{x y}$ from the relation $\tan \tan 2 l_{x y}=-C / A$. The angular velocity of Galactic rotation can be found from the relations $\left(\Omega_{Z}\right)_{R_{0}}=B-A=-M_{12}$. The equations are written in such a form that the negative sign of the angular velocity corresponds to the direction of Galactic rotation.

The matrix (tensor) $M$ can be divided into symmetric, $M^{+}$, and antisymmetric, $M^{-}$, parts. Following Ogorodnikov (1965), we call them the local deformation tensor and the local rotation tensor, respectively:

$$
\begin{gathered}
M_{p q}^{+}=\frac{1}{2}\left(\frac{\partial u_{p}}{\partial r_{q}}+\frac{\partial u_{q}}{\partial r_{p}}\right)_{\circ}, \\
M_{p q}^{-}=\frac{1}{2}\left(\frac{\partial u_{p}}{\partial r_{q}}-\frac{\partial u_{q}}{\partial r_{p}}\right)_{\circ}, \\
(p, q=1,2,3) .
\end{gathered}
$$

Using only the proper motions. When only the stellar proper motions are used, one of the diagonal terms of the local deformation tensor is known to become uncertain. Therefore, we can determine only the differences, for example, $\left(M_{11}^{+}-M_{22}^{+}\right)$and $\left(M_{33}^{+}-M_{22}^{+}\right)$. In this case, we use the conditional equations

$$
\begin{gathered}
4.74 r \mu_{l} \cos b=X_{\odot} \sin l-Y_{\odot} \cos l- \\
-r\left(M_{32}^{-} \cos l \sin b-M_{13}^{-} \sin l \sin b+M_{21}^{-} \cos b+\right. \\
+M_{12}^{+} \cos 2 l \cos b-M_{13}^{+} \sin l \sin b+ \\
\left.+M_{23}^{+} \cos l \sin b-0.5\left(M_{11}^{+}-M_{22}^{+}\right) \sin 2 l \cos b\right) \\
4.74 r \mu_{b}=X_{\odot} \cos l \sin b+Y_{\odot} \sin l \sin b- \\
-Z_{\odot} \cos b+r\left(M_{32}^{-} \sin l-M_{13}^{-} \cos l-\right.
\end{gathered}
$$




$$
\begin{gathered}
-0.5 M_{12}^{+} \sin 2 l \sin 2 b+M_{13}^{+} \cos l \cos 2 b+ \\
+M_{23}^{+} \sin l \cos 2 b-0.5\left(M_{11}^{+}-M_{22}^{+}\right) \cos ^{2} l \sin 2 b+ \\
\left.+0.5\left(M_{33}^{+}-M_{22}^{+}\right) \sin 2 b\right) .
\end{gathered}
$$

$M_{21}^{-}$is equivalent to the Oort constant $B$. In accordance with the chosen coordinate system, the positive directions of rotation are: from axis 1 to 2, from axis 2 to 3 , and from axis 3 to $1 . M_{12}^{+}$is equivalent to the Oort constant A. The diagonal components of the local deformation tensor $M_{11}^{+}, M_{22}^{+}, M_{33}^{+}$(by definition, they coincide with $M_{11}, M_{22}, M_{33}$ ) describe the general contraction or expansion (depending on the sign) of the entire stellar system. Thus, the system of conditional equations (7)-(8) includes eleven sought-for unknowns to be determined by the least squares method.

Although the unknowns $M_{13}^{-}$and $M_{13}^{+}$as well as $M_{32}^{-}$and $M_{23}^{+}$in Eq. (7) being determined cannot be separated between themselves, because they have the same coefficients $(\sin l \sin b$ and $\cos l \sin b$, respectively), all variables can be separated through the simultaneous solution of the system of equations (7) and (8).

The Oort constant $K=\left(M_{11}^{+}+M_{22}^{+}\right) / 2$ can be calculated on the basis of the quantities found by solving the system of equations (7)-(8) as follows:

$$
K=\left(\left(M_{11}^{+}-M_{22}^{+}\right)-2\left(M_{33}^{+}-M_{22}^{+}\right)\right) / 2
$$

by assuming $M_{33}^{+}$to be close to zero. Therefore, we first determine $M_{33}^{+}$by solving the system of equations (3)-(5) based on the sample of RGC stars with known trigonometric parallaxes, radial velocities, and proper motions to test this assumption. We determine the vertex deviation $l_{x y}$ from the relation $\tan 2 l_{x y}=-C / A=-0.5\left(M_{11}^{+}-M_{22}^{+}\right) / M_{12}^{+}$.

\subsection{The Statistical Method}

We use the well-known statistical method (Trumpler and Weaver 1953; Parenago 1951, 1954) to determine the parameters of the residual velocity (Schwarzschild) ellipsoid. It consists in determining the symmetric tensor of moments or the tensor of residual stellar velocity dispersions. When simultaneously using the stellar radial velocities and proper motions to find the six unknown components of the dispersion tensor, we have six equations for each star. The semiaxes of the residual velocity ellipsoid, which we denote by $\sigma_{1,2,3}$ can be determined by analyzing the eigenvalues of the dispersion tensor. We denote the directions of the principal axes of this ellipsoid by $l_{1,2,3}$ and $b_{1,2,3}$.

\section{RESULTS}

The results of solving the system of equations (3)-(5) that we obtained based on the sample of 3632 RGC stars with known trigonometric parallaxes, radial velocities, and proper motions are presented in Table 1. The kinematic parameters were found using both trigonometric parallaxes and photometric distances with the constraints $e_{\pi} / \pi<1$ and $\pi>1$ mas. A preliminary analysis shows that we need these constraints to eliminate the stars that spoil the statistics and affect significantly the parameters being determined.

As we see from Table 1, $M_{33}$ is found to be statistically insignificant. Therefore, we have every reason to neglect $M_{33}$ below when determining the K effect from Eq. (9) based only on the stellar proper motions. 
The parameters of the Ogorodnikov-Milne model obtained on the basis of 95633 RGC stars are presented in Table 2. Based on the results of Gontcharov (2008) and the data of Fig. 1, we may conclude that the spatial distribution of very distant RGC stars is highly nonuniform. Using RGC stars farther than $1 \mathrm{kpc}$ can lead to undesirable selection effects. Moreover, as was shown by Olling and Dehnen (2003), the nonuniformity of the distribution in Galactic longitude affects noticeably the determination of kinematic parameters, in particular, the Oort constants. Therefore, we consider stars closer than $1 \mathrm{kpc}$. Columns nos. 1-3 in Table 2 give the parameters obtained with various constraints on the heliocentric distance of a star $d\left(d_{\text {phot }}\right)$; columns nos. 4 and 5 give the parameters for stars with distances of 500-1000 pc located near the Galactic plane $(|Z|<200 \mathrm{pc})$ and higher $(|Z| \geq 200 \mathrm{pc})$, respectively.

We determined the parameters of the residual velocity ellipsoid based on the statistical method both for all stars of the catalog and for individual samples. The first column in Table 3 gives the parameters of the residual velocity ellipsoid for the sample of 3632 stars considered above. We estimated the errors in the parameters obtained from this sample based on the well-known method by Parenago (1951) using the fourth-order moments.

The complete sample of stars was divided into individual groups by the physical properties of the stars themselves as well as by the heliocentric distance $\mathrm{d}$ and the elevation above the Galactic plane $|Z|$. The results are presented in columns nos. $2-4$ of Table 3 in the former case and in Table 4 and Fig. 2, which shows the residual velocity dispersions as a function of $|Z|$ found by the statistical method using the proper motions of 95633 RGC stars, in the latter case.

We divided the RGC stars by their physical properties based on the selection parameters "Sel1" and "Sel2" listed in the last columns of the catalog by Gontcharov (2008). The first parameter points to the displacement of a star from the center of the distribution of young RGC stars on the $(B-V)_{0}$-reduced proper motion diagram, while the second parameter points to the displacement of a star from a similar diagram for old RGC stars (Gontcharov 2008). We formed three groups: group (1) consists of stars with a difference $0<\Delta S$, where $\Delta S=$ Sel1-Sel2, which includes old normal-metallicity stars; the stars of group (2) have $-0.38<\Delta S<0$ and belong to old low-metallicity stars; finally, group (3) with $-1.0<\Delta S<-0.38$ contains young normal metallicity stars. Consequently, the third and second groups include the youngest and oldest stars, respectively. The increase in average $|Z|$ as we pass from the relatively young groups to the older ones: $\overline{|Z|}_{(3)}=183$ $\mathrm{pc}, \overline{|Z|}_{(1)}=201 \mathrm{pc}, \overline{|Z|}_{(2)}=221 \mathrm{pc}$, indicates that this division identifies the stars by age statistically correctly. Since only the proper motions of RGC stars were used in this case (their space velocities $U, V, W$ cannot be determined), we estimated the errors in the parameters of the residual velocity ellipsoid given in column no. 5 of Table 3 statistically, based on the Monte Carlo method.

Figure 3 shows the distributions of residual UV velocities smoothed by the method described by Bobylev et al. (2006b) for groups (1), (2), and (3) (separated by the parameter ?S); we used only 3632 RGC stars with known trigonometric parallaxes, radial velocities, and proper motions (see Table 1). As we see from the figures, in comparison with sample (3) of "young" stars, the UV distributions for samples (1) and (2) of "old" stars exhibit a larger velocity dispersion manifested as the "spreading" of peaks and the appearance of new clumps, which is caused by the prolonged action of some dynamical factor (a bar, a spiral density wave, and the like). However, it should be noted that the velocity dispersions calculated using the known trigonometric parallaxes rather than the photometric distances 
(as with the complete sample) show the highest values for sample (1), not for (2), which is in conflict with the results of Table. 3. Qualitatively, the older age of the stars from group (1) follows from a comparison of the $U V$-velocity distributions shown in Fig. 3.

Consequently, the method of selection by the parameter $\Delta S$ we used is a reliable method for identifying only the comparatively "young" fraction of stars. A reliable separation of stars with various ages among the remaining "old" fraction of stars is unlikely to be possible, as suggested by the insignificant difference in velocity dispersions for groups (1) and (2), while the velocity dispersions for the young fraction of stars differ significantly. In addition, we obtained contradictory results of the selection by the parameter $\Delta S$ for the entire sample of $\approx 97000$ stars and the sample of 3632 stars with known parallaxes, radial velocities, and proper motions. Clearly, groups (1) and (2) are actually a mixture of stars with various ages.

In Fig. 4, the contraction velocity $K d$ is plotted against the heliocentric distance $d$.

The parameters being determined depend on the division of the original sample into subsamples according to the measured distances. Since all distances contain errors, on average, they show systematic deviations from the true distances. These deviations correlate with the measured distances themselves (the largest measured distances are, on average, overestimated, while the smallest ones are, on average, underestimated).

Therefore, to ascertain the dependence of the kinematic parameters being determined on the distances including the measurement errors, we performed statistical Monte Carlo simulations. To this end, we solved the system of equations (7) and (8) with artificially introduced random errors in the distances distributed normally. The division into subsamples was made by the derived model distances. We analyzed all stars of the catalog without any constraints, but the star was rejected in the case where a negative distance emerged. The equations were solved for 100 realizations for each subsample. We considered three cases: (i) without introducing any errors, so that these results are a good addition to the data of Table 2; (ii) the model errors are $20 \%$ of the measured distance, which is a typical error in the photometric distance; (iii) the model errors are $60 \%$ of the measured distance (considered as the extreme case).

The simulation results are presented in Figs. 5, 6, and 7, where all eleven parameters of the Ogorodnikov-Milne model are plotted against the distance. Figures 5a, 6a, and 7a show the velocity components of the Sun relative to the centroid of the stars under consideration $X_{\odot}, Y_{\odot}, X_{\odot} ;$ Figs. $5 \mathrm{~b}, 6 \mathrm{~b}$, and $7 \mathrm{~b}$ show the parameters $M_{p q}$.

Analysis of these data leads us to conclude that: (a) when stars with large distances are used, a significant bias is observed only for the velocity of the Sun $Y_{\odot}$, while all three velocity components of the Sun are subjected to a similar bias for a large range of specified errors (Fig. 7a); (b) the parameters $M_{p q}$ being determined are smoothed out as the level of model errors increases, which is particularly clearly seen from the middle part of Fig. 7b; (c) it is necessary to reject very close $(d<200-250 \mathrm{pc})$ stars for a reliable determination of all model parameters, except the velocity components of the Sun, which, on the contrary, are determined more reliably from the samples of nearby stars; (d) three parameters are least subjected to any biases: $M_{12}^{+}, M_{21}^{-}$(the Oort constants), and $\left(M_{11}^{+}-M_{22}^{+}\right)$.

We calculated the correlation coefficients (coefficient $k$ ) between the parameters being determined, which arise because the system of equations (7)-(8) is ill-conditioned: (i) $k=$ 0.44 for the pair $\left(M_{23}^{+}\right)-\left(M_{32}^{-}\right)$, (ii) $k=0.41$ for the pair $\left(M_{33}^{+}-M_{22}^{+}\right)-\left(M_{11}^{+}-M_{22}^{+}\right)$, (iii) for the sample of most distant stars (Figs. 5-7), $k=0.37$ for the pair $\left(M_{12}^{+}\right)-\left(M_{21}^{-}\right)$, while for closer stars, the correlation coefficient $k$ is nearly zero for this pair. The correlations between the velocity $Y_{\odot}$, which is subjected to the largest bias, and all the remaining model 
parameters are nearly zero.

The slight increase in velocity $Y_{\odot}$ with distance that we see in Fig. 5a can be explained by an asymmetric drift (Bobylev and Bajkova 2007). Indeed, the mean value of $\Delta S$ changes gradually from $0.1(\bar{d}=150 \mathrm{pc})$ to $-0.1(\bar{d}=900 \mathrm{pc})$.

\section{DISCUSSION}

Galactic Rotation Parameters As can be seen from the first column of Table 2, the Oort constants are: $A=15.9 \pm 0.2 \mathrm{~km} \mathrm{~s}^{-1} \mathrm{kpc}^{-1}$ and $B=-12.0 \pm 0.2 \mathrm{~km} \mathrm{~s}^{-1} \mathrm{kpc}^{-1}$. Based on these parameters, we estimate the circular velocity of rotation of the solar neighborhood to be $V_{0}=\left|R_{0} \Omega_{Z}\right|=222 \pm 2 \mathrm{~km} \mathrm{~s}^{-1}$ for $R_{0}=8.0 \mathrm{kpc}$ and the period of its revolution around the Galactic center to be 221 Myr.

The Oort constants found are in good agreement with their determinations in other works. For example, using data on young objects of the Galactic disk, Bobylev et al. (2008) found $A=15.5 \pm 0.3 \mathrm{~km} \mathrm{~s}^{-1} \mathrm{kpc}^{-1}$ and $B=-12.2 \pm 0.7 \mathrm{~km} \mathrm{~s}^{-1} \mathrm{kpc}^{-1}$.

\subsection{Parameters of the Velocity Ellipsoid}

We clearly see from Table 2 that the kinematic parameters differ for different groups of giants located near the Galactic plane at $|Z|<200$ pc (column 4) and at large elevations above the Galactic plane $|Z| \geq 200 \mathrm{pc}$ (column 5). The vertex deviation reaches $l_{x y}=9.1 \pm 0^{\circ} .5$ for the first group and is a factor of 2 smaller, $4.9 \pm 0^{\circ} .6$, for the second group. Note that the difference in kinematic parameters is enhanced with increasing elevation above the Galactic plane. On the whole, our results are in agreement with those of Rybka (2008).

From Table 3, we see an increase in velocity dispersions and a decrease in vertex deviation with increasing "age" of the sample of stars. Column no. 1 gives the parameters obtained for stars with available radial velocities from all three space velocity components. Using the radial velocities affects the determination of the parameters of the velocity ellipsoid, with the most prominent parameter being $l_{1}=11^{\circ} .7$. For other samples, there is also a difference in vertex deviation. Thus, for example, $l_{1}=5^{\circ} .1$ for the sample of oldest stars is considerably smaller than $l_{1}=9^{\circ} .4$ for the youngest group.

On the other hand, since RGC stars with various ages are well mixed in space, using various constraints on the spatial coordinates of stars does not lead to any noticeable change in the parameters of the velocity ellipsoid (Table 4).

In contrast to the approach used by Rybka (2008), we traced the changes in the parameters of the velocity ellipsoid with $|Z|$ (Fig. 2). As expected, our results show that younger stars with a lower velocity dispersion are concentrated to the Galactic plane to a greater extent.

\subsection{The Contraction Effect}

According to Table 2, the Oort constant $\mathrm{K}$ reaches its maximum value for the sample of distant (500-1000 pc, $d=0.65 \mathrm{kpc}$, column no. 4) RGC stars located near the Galactic plane $(|Z|<200 \mathrm{pc}),-5.3 \pm 1.3 \mathrm{~km} \mathrm{~s}^{-1} \mathrm{kpc}^{-1}$, while the contraction velocity is $K d=-3.5 \pm 0.9$ $\mathrm{km} \mathrm{s}^{-1}$; this value is indicated in Fig. 4 by the filled circle. The open circles in Fig. 4 indicate five values of $K d$ from the cited data for which estimates of the average distance are 
available. Below, we list them, as denoted by the numbers in Fig. 4, in order of increasing d:

(1) the contraction velocity determined from the space velocities of A0-A5 giants (Bobylev et al. 2006b) for which $K=-13.1 \pm 2.0 \mathrm{~km} \mathrm{~s}^{-1} \mathrm{kpc}^{-1}$ was obtained, with $d=0.19$ $\mathrm{kpc}$;

(2) the estimate from Rybka (2004b) obtained using a sample of $\approx 11000$ Tycho- 2 G5-K0 giants with $|b|<50^{\circ}, d>250 \mathrm{pc}, d=0.364 \mathrm{kpc}$;

(3) the estimate from Rybka (2004a) obtained using a sample of ? 9000 main-sequence Tycho-2 B0-B9 stars, $d=0.53 \mathrm{kpc}$;

(4) the estimate from Rybka (2008) obtained using $\approx 30000$ Tycho-2 RGC stars at $|b|<$ $30^{\circ}$ and $d>100 \mathrm{pc}$ for which we found the average photometric distance based on the catalog by Gontcharov (2008), $d=0.54 \mathrm{kpc}$;

(5) the result obtained using open star clusters with an average age of $\approx 100$ Myr (Bobylev et al. 2007) at $d=1.05 \mathrm{kpc}$.

Note also the result of analyzing OB stars located in the range of distances 600-2000 pc from Torra et al. (2000) for which $K=-2.9 \pm 0.6 \mathrm{~km} \mathrm{~s}^{-1} \mathrm{kpc}^{-1}$ was found. Taking $\bar{d} \approx 0.8 \mathrm{kpc}$ as a realistic average distance for these stars (which is not given in Torra et al. (2000)), we will obtain $K d=-2.3 \mathrm{~km} \mathrm{~s}^{-1}$, a value consistent with the data in Fig. 4. A comprehensive overview of the results of determining the negative $\mathrm{K}$ effect by other authors can be found in Fernández et al. (2001).

As can be seen from Fig. 4, on the whole, there is good agreement between the various contraction velocity estimates. The main purpose of our comparison is to show that the results of different authors obtained by different methods agree in sign, i.e., the contraction effect actually takes place, and, given the errors, they also agree satisfactorily in magnitude. However, the difference for the A0-A5 giants of the OSACA database (Bobylev et al. 2006b) is most pronounced. This difference can be explained by the fact that the sample of A0-A5 stars was specially formed by a kinematic indicatorby the maximum $\mathrm{K}$ effect. At the same time, this result is in good agreement with the data of Table 1, according to which we have $K d=-1.8 \pm 0.5 \mathrm{~km} \mathrm{~s}^{-1}$.

What is the maximum contraction velocity? In the opinion of Rybka (2004a), $K d=$ $-8.2 \pm 0.9 \mathrm{~km} \mathrm{~s}^{-1}$ at $d=1 \mathrm{kpc}$. However, this estimate was obtained by extrapolating the data on main-sequence dwarfs $(d<0.6 \mathrm{kpc})$. Analysis of the kinematics of open star clusters (Bobylev et al. 2007) showed the magnitude of the velocity Kd to decrease at $d>1 \mathrm{kpc}$.

Note also the ambiguity of treating $K=0.5\left(M_{11}+M_{22}\right)$ as the effect of contraction in the plane. Indeed, as we see from Table $1, M_{22}=\partial u_{2} / \partial r_{2}$ does not differ significantly from zero, with large values of $K$ and $l_{x y}$ being reached solely through the gradient $M_{11}=\partial u_{1} / \partial r_{1}$. Thus, we can only assert that noticeable deviations from circular motions are observed. The nature of these deviations requires a further study.

\subsection{The Problem of the Galactic Disk Warp}

Rotation around the $\mathbf{X}$ axis. As can be seen from Table 2 (the third column), $M_{32}^{-}=$ $-1.4 \pm 0.2 \mathrm{~km} \mathrm{~s}^{-1} \mathrm{kpc}^{-1}$ and $M_{23}^{+}=+1.1 \pm 0.3 \mathrm{~km} \mathrm{~s}^{-1} \mathrm{kpc}^{-1}$. The negative sign of $M_{32}^{-}$means that the rotation is from the $Z$ axis to the $Y$ axis, i.e., the stars approach to the Galactic plane. They approach from the North Pole in the first and second quadrants and from the South Pole in the third and fourth quadrants. By analogy with the rotation around the 
$Z\left(\Omega_{Z}=B-A\right)$ axis, the angular velocity of rotation around the $X$ axis can be determined as $\Omega_{X}=M_{32}^{-}-M_{23}^{+}=-2.5 \pm 0.3 \mathrm{~km} \mathrm{~s}^{-1} \mathrm{kpc}^{-1}$.

This is inconsistent with the analysis of the motions of $\approx 4000$ giants of various spectral types O-M performed by Miyamoto et al. (1993), who found a positive direction of rotation around the $X$ axis (directed toward the Galactic center). Note that, in this case, the stellar proper motions being analyzed were determined in the FK5 system, which is noticeably distorted by the uncertainty in the precession constant. On the other hand, having analyzed the proper motions of O-B5 stars with the Hipparcos (19977) system, Miyamoto and Zhu (1998) also reached the conclusion about a positive rotation around the $X$ axis. In our notation, Miyamoto and Zhu (1998) obtained $\Omega_{X}=2 M_{32}^{-} \equiv 2 M_{23}^{+}=+3.8 \pm 1.1 \mathrm{~km} \mathrm{~s}^{-1}$ $\mathrm{kpc}^{-1}$. However, it should be noted that Miyamoto et al. (1993) and Miyamoto and Zhu (1998) used a model where the variables $M_{32}^{-}$and $M_{23}^{+}$could not be separated and, therefore, they were assumed to be equivalent. In our approach, which consists in solving Eqs. (7)-(8), we can separate these variables. This is possible, because the distribution of RGC stars over the celestial sphere is fairly uniform.

The parameters found with a constraint on $|Z|$ and given in column no. 4 of Table 2 are also of great interest. As we see, for the sample of stars close to the Galactic plane (this case corresponds largely to the distribution of $\mathrm{O}-\mathrm{B} 5$ stars), the solutions for $\mathrm{M}$ with index 3 differ markedly from those obtained for the samples filling uniformly the celestial sphere (Table 2, columns nos. 1, 2, 3, 5).

As can be seen from Table 2 and Fig. 5, the values of the parameters $M_{32}^{-}$and $M_{23}^{+}$do not depend strongly on the distance. However, the motions of nearby stars can be distorted by local peculiarities (in particular, by the kinematic peculiarities of the Gould Belt with a characteristic radius of about $500 \mathrm{pc}$ ). Therefore, the values of these parameters determined from distant stars seem more reliable. Thus, we interpret $\Omega_{X}$ found from relatively distant RGC stars as the rotation related to the warp of the Galactic stellar-gaseous disk.

Note that owing to the use of currently available data that realize the inertial frame of reference more accurately than FK5 and the possibility of separating the components $M_{32}^{-}$ and $M_{23}^{+}$when solving Eqs. (7)-(8), we have every reason to believe that our results are more reliable than those of Miyamoto et al. (1993) and Miyamoto and Zhu (1998).

Rotation around the $\mathbf{Y}$ axis. There is a large uncertainty with regard to the magnitude and direction of the rotation around the $\mathrm{Y}$ axis related to the disk warp. Thus, for example, having analyzed the motions of $\approx 4000$ giants of various spectral types $\mathrm{O}$ M, Miyamoto et al. (1993) found a positive direction of rotation around the $Y$ axis: $\Omega_{Y}=2 M_{13}^{-} \equiv-2 M_{13}^{+}=+5.6 \pm 1.0 \mathrm{~km} \mathrm{~s}^{-1} \mathrm{kpc}^{-1}$. Based on an analysis of the Hipparcos data, Drimmel et al. (2000) obtained a completely different estimate in the form of precession of $\mathrm{OB}$ stars in the $Z X$ plane with an angular velocity of $-25 \mathrm{~km} \mathrm{~s} \mathrm{kpc}^{-1}$ (i.e., rotation around the $Y$ axis). However, Bobylev (2004) showed that this value could be explained by inaccurate referencing of the ICRS/Hipparcos realization to the system of extragalactic sources.

Based on the data of column no. 2 in Table 2, we have $\Omega_{Y}=M_{13}^{-}-M_{13}^{+}=-1.5 \pm 0.3 \mathrm{~km} \mathrm{~s}^{-1}$ $\mathrm{kpc}^{-1}$. As we see, the value of this component is determined almost entirely by $M_{13}^{-}$. In this respect, $M_{13}^{-} / 4.74=-0.41 \pm 0.05 \mathrm{mas} \mathrm{yr}^{-1}$, which is in excellent agreement with the residual rotation of the ICRS relative to the inertial frame of reference, $M_{13}^{-}=-0.37 \pm 0.04 \mathrm{mas} \mathrm{yr}^{-1}$, estimated by Bobylev and Khovrichev (2006) based on the Tycho-2 and UCAC2 (Zacharias 2004) Catalogues, is of great interest. Therefore, we cannot say what the contribution related to the disk warp is in this case, since these two effects cannot be separated. Obviously, if we 
apply the correction $M_{13}^{-} \approx-0.4$ mas $\mathrm{yr}^{-1}$ (reducing the Hipparcos system to the inertial frame of reference), then the "remainder" of the rotation around $Y$ axis will be close to zero.

On the whole, we may conclude that the kinematic effect related to the disk warp manifests itself mainly as the rotation around the $X$ axis (see the previous section). The small magnitude of the rotation velocity around the $Y$ axis is in agreement with the fact that the line of nodes of the HI layer is known to be close to the direction of the Galactic center/anticenter.

\section{CONCLUSIONS}

We analyzed the proper motions of 95633 RGC stars from the Tycho-2 Catalogue using the linear Ogorodnikov-Milne model. The photometric distances to these stars were estimated by Gontcharov (2008) based on 2MASS photometric data.

Based on a subsample of 3632 RGC stars for which not only the proper motions but also the radial velocities, photometric distances, and Hipparcos trigonometric parallaxes are available, we showed that, apart from the star centroid velocity components relative to the Sun $\left(X_{\odot}, Y_{\odot}, Z_{\odot}\right)$, only those parameters of the Ogorodnikov-Milne model that describe the stellar motions in the $X Y$ plane differ significantly from zero. This allowed us to study the plane $K$ effect based the tangential velocities of a considerably larger sample of RGC stars.

To ascertain the dependence of the kinematic parameters being determined on the distances, including the measurement errors in the distances, we performed statistical Monte Carlo simulations. Our simulations showed that, first, a significant bias is observed only for the velocity $Y_{\odot}$ at large distances and, second, very close $(d<200-250 \mathrm{pc})$ stars should be excluded to reliably determine all parameters of the Ogorodnikov-Milne model, except the velocity components of the Sun, which, on the contrary, are determined more reliably from samples of nearby stars.

Analysis of the entire sample of RGC stars showed that the Oort constants that describe the rotation around the $\mathrm{Z}$ axis are $A=15.9 \pm 0.2 \mathrm{~km} \mathrm{~s}^{-1} \mathrm{kpc}^{-1}$ and $B=-12.0 \pm 0.2 \mathrm{~km} \mathrm{~s}^{-1}$ $\mathrm{kpc}^{-1}$. This gives an estimate of the rotation velocity of the solar neighborhood, $V_{0}=222 \pm 2$ $\mathrm{km} \mathrm{s}^{-1}$, for $R_{0}=8.0 \mathrm{kpc}$ and the period of its revolution around the Galactic center, 221 Myr.

Judging by the velocity dispersions found, the RGC sample includes stars of various ages. We made an attempt to roughly separate the RGC stars by ages based on the parameter $\Delta S$. It showed that only the fraction of relatively young RGC stars, which accounts for about $20 \%$ of the entire sample, is identified most reliably.

The most interesting results were obtained using stars located in the range of distances 500-1000 pc. The RGC stars located near the Galactic plane were shown to have a noticeable contraction effect (observed in the XY plane) that depends on the heliocentric distances of the stars. Thus, for example, for the sample of RGC stars located near the Galactic plane $(|Z|<200 \mathrm{pc})$, the contraction velocity is $K d=-3.5 \pm 0.9 \mathrm{~km} \mathrm{~s}^{-1}$ and the vertex deviation is $l_{x y}=9.1 \pm 0^{\circ} .5$. For stars located well above the Galactic plane $(|Z| \geq 200 \mathrm{pc})$, these effects are less pronounced, being $K d=-1.7 \pm 0.5 \mathrm{~km} \mathrm{~s}^{-1}$ and $l_{x y}=4.9 \pm 0^{\circ} .6$.

Based on RGC stars with distances in the range 500-1000 pc, we found the angular velocity of rotation around the Galactic $X$ axis to be $-2.5 \pm 0.3 \mathrm{~km} \mathrm{~s}^{-1} \mathrm{kpc}^{-1}$, which we interpret as the rotation related to the warp of the Galactic stellar-gaseous disk. The direction of this rotation shows that the stars approach the Galactic plane. 


\section{ACKNOWLEDGMENTS}

We are grateful to the referees for helpful remarks that contributed to a significant improvement of the paper. This work was supported by the Russian Foundation for Basic Research (project no. 08-02-00400) and in part by the "Origin and Evolution of Stars and Galaxies" Program of the Presidium of the Russian Academy of Sciences and the Program for State Support of Leading Scientific Schools of Russia (NSh-6110.2008.2 "Multiwavelength Astrophysical Research").

\section{REFERENCES}

1. W.B. Burton, Galactic and Extragalactic Radio Astronomy, Ed. by G. Verschuur and K. Kellerman (Springer, New York, 1988).

2. V.V. Bobylev, Pisma Astron. Zh. 30, 185 (2004) [Astron. Lett. 30, 159 (2004)].

3. V.V. Bobylev and A. T. Bajkova, Astron. Zh. 84, 418 (2007) [Astron. Rep. 51, 372 (2007)].

4. V.V. Bobylev, A.T. Bajkova, and G.A. Gontcharov, Astron. Astrophys. Trans. 25, 143 (2006a).

5. V.V. Bobylev, A.T. Bajkova, S.V. Lebedeva, Pisma Astron. Zh. 33, 809 (2007) [Astron. Lett. 33, 720 (2007)].

6. V.V. Bobylev, A.T. Bajkova, and A.S. Stepanishchev, Pisma Astron. Zh. 34, 570 (2008) [Astron. Lett. 34, 515 (2008)].

7. V.V. Bobylev, G.A. Gontcharov, and A.T. Bajkova, Astron. Zh. 83, 821 (2006b) [Astron. Rep. 50, 733 (2006)].

8. V.V. Bobylev and M. Yu. Khovrichev, Pisma Astron. Zh. 32, 676 (2006) [Astron. Lett. 32, 608 (2006)].

9. S.V.M. Clube, Mon. Not. R. Astron. Soc. 159, 289 (1972).

10. S.V.M. Clube, Mon. Not. R. Astron. Soc. 161, 445 (1973).

11. W. Dehnen, Astron. J. 115, 2384 (1998).

12. R. Drimmel, R.L. Smart, and M.G. Lattanzi, Astron. Astrophys. 354, 67 (2000).

13. R. Drimmel and D.N. Spergel, Astroph. J. 556, 181 (2001).

14. D. Fernández, F. Figueras, and J. Torra, Astron. Astrophys. 372, 833 (2001).

15. G.A. Gontcharov, Pisma Astron. Zh. 32, 844 (2006) [Astron. Lett. 32, 759 (2006)].

16. G.A. Gontcharov, Pisma Astron. Zh. 34, 868 (2008) [Astron. Lett. 34, 785 (2008)].

17. The HIPPARCOS and Tycho Catalogues, ESA SP- 1200 (1997).

18. E. Hog, C. Fabricius, V.V. Makarov, et al., Astron. Astrophys. 355, L27 (2000).

19. P.M.W. Kalberla and L. Dedes, Astron. Astrophys. 487, 951 (2008).

20. M.R. Metzger, J.A.R. Caldwell and P.L. Schechter, Astron. J. 115, 635 (1998).

21. M. Miyamoto, M. Soma, and M. Yoshizawa, Astron. J. 105, 2138 (1993).

22. M. Miyamoto and Z. Zhu, Astron. J. 115, 1483 (1998).

23. K.F. Ogorodnikov, Dynamics of Stellar Systems (Fizmatgiz, Moscow, 1965) [in Russian].

24. R.P. Olling and W. Dehnen, Astroph. J. 599, 275 (2003).

25. P.P. Parenago, Tr. GAISh 20, 26 (1951).

26. P.P. Parenago, A Course on Stellar Astronomy (Gosizdat, Moscow, 1954) [in Russian].

27. F. Pont, M. Mayor, and G. Burki, Astron. Astrophys. 285, 415 (1994).

28. K. Rohlfs, Lectures in Density Waves (Springer, Berlin, 1977). 
29. S.P. Rybka, Kinem. Fiz. Neb. Tel 20, 133 (2004a).

30. S.P. Rybka, Kinem. Fiz. Neb. Tel 20, 437 (2004b).

31. S.P. Rybka, Kinem. Fiz. Neb. Tel 22, 225 (2006).

32. S.P. Rybka, Kinem. Fiz. Neb. Tel 23, 102 (2007) [Kin. Phys. Cel. Bodies 23, 70 (2007)].

33. S.P. Rybka, Kinem. Fiz. Neb. Tel 24, 137 (2008) [Kin. Phys. Cel. Bodies 23, 99 (2008)].

34. M.F. Skrutskie, R.M. Cutri, R. Stiening, et al., Astron. J. 131, 1163 (2006).

35. M. Steinmetz, T. Zwitter, A. Seibert, et al., Astron. J. 132, 1645 (2006).

36. J. Torra, D. Fernández, and F. Figueras, Astron. Astrophys. 359, 82 (2000).

37. R.J. Trumpler and H.F. Weaver, Statistical Astronomy (Univ. of Calif., Berkely, 1953).

38. I. Yusifov, astro-ph: 0405517 (2004).

39. N. Zacharias, S.E. Urban, M.I. Zacharias, et al., Astron. J. 127, 3043 (2004). 
Table 1: Kinematic parameters obtained by solving the system of equations (3)-(5)

\begin{tabular}{|r|r|r|}
\hline$N_{\star}$ & 3632 & \multicolumn{1}{|c|}{3632} \\
\hline & 1 & \multicolumn{1}{|c|}{2} \\
\hline$X_{\odot}, \mathrm{km} \mathrm{s}^{-1}$ & $9.9 \pm 0.4$ & $9.5 \pm 0.4$ \\
$Y_{\odot}, \mathrm{km} \mathrm{s}^{-1}$ & $16.8 \pm 0.4$ & $16.3 \pm 0.4$ \\
$Z_{\odot}, \mathrm{km} \mathrm{s}^{-1}$ & $7.3 \pm 0.4$ & $6.9 \pm 0.4$ \\
$M_{11}, \mathrm{~km} \mathrm{~s}^{-1} \mathrm{kpc}^{-1}$ & $-15.5 \pm 3.3$ & $-14.3 \pm 3.7$ \\
$M_{12}, \mathrm{~km} \mathrm{~s}^{-1} \mathrm{kpc}^{-1}$ & $28.7 \pm 3.2$ & $29.3 \pm 3.5$ \\
$M_{13}, \mathrm{~km} \mathrm{~s}^{-1} \mathrm{kpc}^{-1}$ & $-2.8 \pm 2.6$ & $0.6 \pm 2.9$ \\
$M_{21}, \mathrm{~km} \mathrm{~s}^{-1} \mathrm{kpc}^{-1}$ & $8.6 \pm 3.3$ & $5.9 \pm 3.7$ \\
$M_{22}, \mathrm{~km} \mathrm{~s}^{-1} \mathrm{kpc}^{-1}$ & $-1.3 \pm 3.2$ & $-2.2 \pm 3.5$ \\
$M_{23}, \mathrm{~km} \mathrm{~s}^{-1} \mathrm{kpc}^{-1}$ & $-5.8 \pm 2.6$ & $-3.8 \pm 2.9$ \\
$M_{31}, \mathrm{~km} \mathrm{~s}^{-1} \mathrm{kpc}^{-1}$ & $-6.7 \pm 3.3$ & $-4.3 \pm 3.7$ \\
$M_{32}, \mathrm{~km} \mathrm{~s}^{-1} \mathrm{kpc}^{-1}$ & $-5.3 \pm 3.2$ & $-4.1 \pm 3.5$ \\
$M_{33}, \mathrm{~km} \mathrm{~s}^{-1} \mathrm{kpc}^{-1}$ & $-1.1 \pm 2.6$ & $-4.3 \pm 2.9$ \\
$A, \mathrm{~km} \mathrm{~s}^{-1} \mathrm{kpc}^{-1}$ & $18.6 \pm 2.3$ & $17.6 \pm 2.5$ \\
$B, \mathrm{~km} \mathrm{~s}^{-1} \mathrm{kpc}^{-1}$ & $-10.0 \pm 2.3$ & $-11.7 \pm 2.5$ \\
$C, \mathrm{~km} \mathrm{~s}^{-1} \mathrm{kpc}^{-1}$ & $-7.1 \pm 2.3$ & $-6.1 \pm 2.5$ \\
$K, \mathrm{~km} \mathrm{~s}^{-1} \mathrm{kpc}^{-1}$ & $-8.4 \pm 2.3$ & $-8.2 \pm 2.5$ \\
$\bar{d}, \mathrm{kpc}$ & 0.22 & 0.22 \\
\hline
\end{tabular}

Note. The parameters presented in columns nos. 1 and 2 were obtained using trigonometric parallaxes and photometric distances, respectively; $N_{\star}$ is the number of sample stars. 
Table 2: Kinematic parameters obtained by solving the system of equations (7)-(8)

\begin{tabular}{|c|c|c|c|c|c|}
\hline$N_{\star}$ & $\begin{array}{r}d<1000 \mathrm{pc} \\
95633\end{array}$ & $\begin{array}{r}500-1000 \mathrm{pc} \\
50595\end{array}$ & $\begin{array}{r}700-1000 \mathrm{pc} \\
19200\end{array}$ & $\begin{array}{c}500-1000 \mathrm{pc} \\
|Z|<200 \mathrm{pc} \\
22684\end{array}$ & $\begin{array}{c}500-1000 \mathrm{pc} \\
|Z| \geq 200 \mathrm{pc} \\
27911\end{array}$ \\
\hline & 1 & 2 & 3 & 4 & 5 \\
\hline$X_{\odot}, \mathrm{km} \mathrm{s}^{-1}$ & $7.84 \pm 0.09$ & $8.55 \pm 0.13$ & $8.67 \pm 0.22$ & $8.49 \pm 0.18$ & $8.52 \pm 0.18$ \\
\hline$Y_{\odot}, \mathrm{km} \mathrm{s}^{-1}$ & $15.94 \pm 0.10$ & $17.74 \pm 0.14$ & $19.19 \pm 0.26$ & $16.14 \pm 0.23$ & $18.48 \pm 0.19$ \\
\hline$Z_{\odot}, \mathrm{km} \mathrm{s}^{-1}$ & $6.60 \pm 0.08$ & $7.17 \pm 0.12$ & $7.59 \pm 0.21$ & $6.86 \pm 0.15$ & $7.59 \pm 0.19$ \\
\hline$M_{21}^{+}(A), \mathrm{km} / \mathrm{s} / \mathrm{kpc}$ & $15.87 \pm 0.20$ & $15.86 \pm 0.24$ & $15.61 \pm 0.37$ & $16.25 \pm 0.35$ & $15.33 \pm 0.35$ \\
\hline$M_{32}^{-}, \mathrm{km} / \mathrm{s} / \mathrm{kpc}$ & $-1.40 \pm 0.17$ & $-1.41 \pm 0.21$ & $-1.80 \pm 0.31$ & $1.83 \pm 0.95$ & $-1.70 \pm 0.26$ \\
\hline$M_{13}^{-}, \mathrm{km} / \mathrm{s} / \mathrm{kpc}$ & $-2.01 \pm 0.18$ & $-1.93 \pm 0.22$ & $-1.97 \pm 0.32$ & $-5.28 \pm 0.77$ & $-1.70 \pm 0.27$ \\
\hline$M_{21}^{-}(B), \mathrm{km} / \mathrm{s} / \mathrm{kpc}$ & $-11.99 \pm 0.15$ & $-11.99 \pm 0.18$ & $-12.18 \pm 0.26$ & $-11.82 \pm 0.24$ & $-12.26 \pm 0.27$ \\
\hline$M_{11-22}^{+}, \mathrm{km} / \mathrm{s} / \mathrm{kpc}$ & $-7.86 \pm 0.38$ & $-7.43 \pm 0.45$ & $-6.47 \pm 0.68$ & $-10.73 \pm 0.62$ & $-5.29 \pm 0.66$ \\
\hline$M_{13}^{+}, \mathrm{km} / \mathrm{s} / \mathrm{kpc}$ & $-0.45 \pm 0.23$ & $-0.47 \pm 0.27$ & $0.15 \pm 0.40$ & $-3.90 \pm 0.82$ & $-0.31 \pm 0.38$ \\
\hline$M_{23}^{+}, \mathrm{km} / \mathrm{s} / \mathrm{kpc}$ & $0.98 \pm 0.22$ & $1.13 \pm 0.26$ & $1.49 \pm 0.38$ & $-1.79 \pm 1.01$ & $0.88 \pm 0.36$ \\
\hline$M_{33-22}^{+}, \mathrm{km} / \mathrm{s} / \mathrm{kpc}$ & $-1.36 \pm 0.43$ & $-0.99 \pm 0.51$ & $-0.47 \pm 0.75$ & $-0.02 \pm 1.27$ & $-0.27 \pm 0.61$ \\
\hline $\bar{d}, \mathrm{kpc}$ & $0.52 \pm 0.21$ & $0.68 \pm 0.14$ & $0.81 \pm 0.11$ & $0.65 \pm 0.12$ & $0.70 \pm 0.15$ \\
\hline$C, \mathrm{~km} \mathrm{~s}^{-1} \mathrm{kpc}^{-1}$ & $-3.9 \pm 0.2$ & $-3.7 \pm 0.2$ & $-3.2 \pm 0.3$ & $-5.4 \pm 0.3$ & $-2.7 \pm 0.3$ \\
\hline$K, \mathrm{~km} \mathrm{~s}^{-1} \mathrm{kpc}^{-1}$ & $-2.6 \pm 0.5$ & $-2.7 \pm 0.6$ & $-2.8 \pm 0.8$ & $-5.3 \pm 1.3$ & $-2.4 \pm 0.7$ \\
\hline$K \cdot d, \mathrm{~km} \mathrm{~s}^{-1}$ & $-1.4 \pm 0.3$ & $-1.8 \pm 0.3$ & $-2.3 \pm 0.7$ & $-3.5 \pm 0.9$ & $-1.7 \pm 0.5$ \\
\hline$l_{x y}$, deg. & $7.0 \pm 0.3$ & $6.6 \pm 0.4$ & $5.9 \pm 0.6$ & $9.1 \pm 0.5$ & $4.9 \pm 0.6$ \\
\hline
\end{tabular}


Table 3: Parameters of the residual velocity ellipsoid

\begin{tabular}{|c|c|c|c|c|c|}
\hline$N_{\star}$ & 3632 & 39979 & 36529 & 20497 & $\varepsilon$ \\
\hline & 1 & 2 & 3 & 4 & 5 \\
\hline$\sigma_{1}$ & $32.1 \pm 0.4$ & 29.1 & 35.6 & 19.7 & 0.50 \\
$\sigma_{2}$ & $21.2 \pm 0.4$ & 21.7 & 25.9 & 13.1 & 0.40 \\
$\sigma_{3}$ & $17.9 \pm 0.3$ & 16.8 & 20.6 & 10.8 & 0.25 \\
$l_{1}$ & $11^{\circ} .7 \pm 4^{\circ} .0$ & $6^{\circ} .3$ & $5^{\circ} .1$ & $9^{\circ} .4$ & $0^{\circ} .4$ \\
$l_{2}$ & $101^{\circ} .7 \pm 1^{\circ} .4$ & $96^{\circ} .3$ & $91^{\circ} .8$ & $100^{\circ} .1$ & $0^{\circ} .4$ \\
$l_{3}$ & $287^{\circ} .6 \pm 1^{\circ} .4$ & $260^{\circ}$ & $253^{\circ}$ & $265^{\circ}$ & $15^{\circ}$ \\
$b_{1}$ & $-4^{\circ} .1 \pm 0^{\circ} .8$ & $1^{\circ} .0$ & $0^{\circ} .8$ & $3^{\circ} .0$ & $0^{\circ} .2$ \\
$b_{2}$ & $3^{\circ} .9 \pm 0^{\circ} .8$ & $3^{\circ} .6$ & $5^{\circ} .0$ & $11^{\circ} .9$ & $0^{\circ} .6$ \\
$b_{3}$ & $86^{\circ} .1 \pm 1^{\circ} .8$ & $86^{\circ} .3$ & $87^{\circ} .9$ & $77^{\circ} .7$ & $0^{\circ} .5$ \\
\hline
\end{tabular}

Note. Columns nos. 1 and $2-4$ give the parameters determined from the stellar space velocities and only from the stellar proper motions, respectively; column no. 2 for $0<\Delta S$, column no. 3 for $-0.38<\Delta S<0$, column no. 4 for $-1<\Delta S<-0.38$; column no. 5 gives a Monte Carlo estimate of the error $\varepsilon$.

Table 4: Parameters of the residual velocity ellipsoid obtained using the proper motions of RGC stars for the samples of Table 2

\begin{tabular}{|r|r|r|r|r|r|}
\hline & $d<1000 \mathrm{pc}$ & $500-1000 \mathrm{pc}$ & $700-1000 \mathrm{pc}$ & $\begin{array}{r}500-1000 \mathrm{pc} \\
|Z|<200 \mathrm{pc} \\
N_{\star}\end{array}$ & $\begin{array}{r}500-1000 \mathrm{pc} \\
|Z| \geq 200 \mathrm{pc} \\
25911\end{array}$ \\
\hline & 1 & 50595 & 19200 & 4 & 5 \\
\hline$\sigma_{1}$ & 30.1 & 2 & 3 & 4 & 33.0 \\
$\sigma_{2}$ & 21.7 & 32.2 & 35.0 & 31.4 & 24.2 \\
$\sigma_{3}$ & 17.2 & 18.7 & 25.3 & 20.3 & 20.4 \\
$l_{1}$ & $6^{\circ} .0$ & $5^{\circ} .2$ & $5^{\circ} .5$ & 17.3 & $3^{\circ} .8$ \\
$l_{2}$ & $96^{\circ} .0$ & $95^{\circ} .2$ & $95^{\circ} .5$ & $96^{\circ} .6$ & $93^{\circ} .8$ \\
$l_{3}$ & $257^{\circ} .9$ & $91^{\circ} .1$ & $83^{\circ} .7$ & $242^{\circ} .7$ & $80^{\circ} .1$ \\
$b_{1}$ & $0^{\circ} .7$ & $-3^{\circ} .0$ & $-1^{\circ} .2$ & $0^{\circ} .5$ & $-1^{\circ} .1$ \\
$b_{2}$ & $2^{\circ} .3$ & $-4^{\circ} .2$ & $-5^{\circ} .7$ & $0^{\circ} .7$ & $-4^{\circ} .6$ \\
$b_{3}$ & $87^{\circ} .6$ & $85^{\circ} .8$ & $84^{\circ} .2$ & $89^{\circ} .1$ & $85^{\circ} .3$ \\
\hline
\end{tabular}



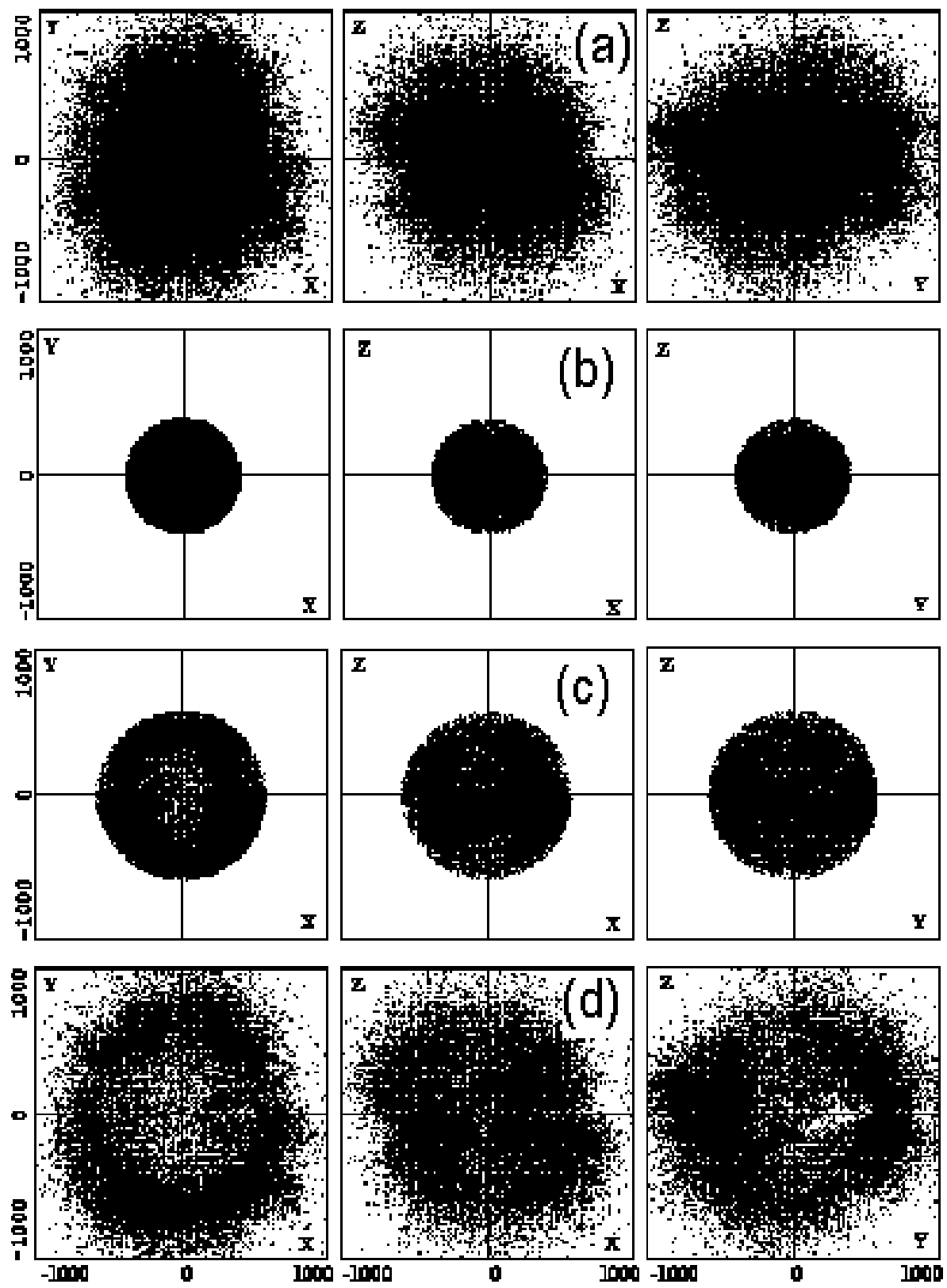

Fig. 1. Distributions of the samples of RGC stars in the $X Y, X Z$, and $Y Z$ planes (the left, central, and right columns, respectively): the complete sample (a), the stars with distances $d<400 \mathrm{pc}(\mathrm{b}), 400 \mathrm{pc}<d<600 \mathrm{pc}(\mathrm{c})$, and $600 \mathrm{pc}<d<1600 \mathrm{pc}(\mathrm{d})$. 


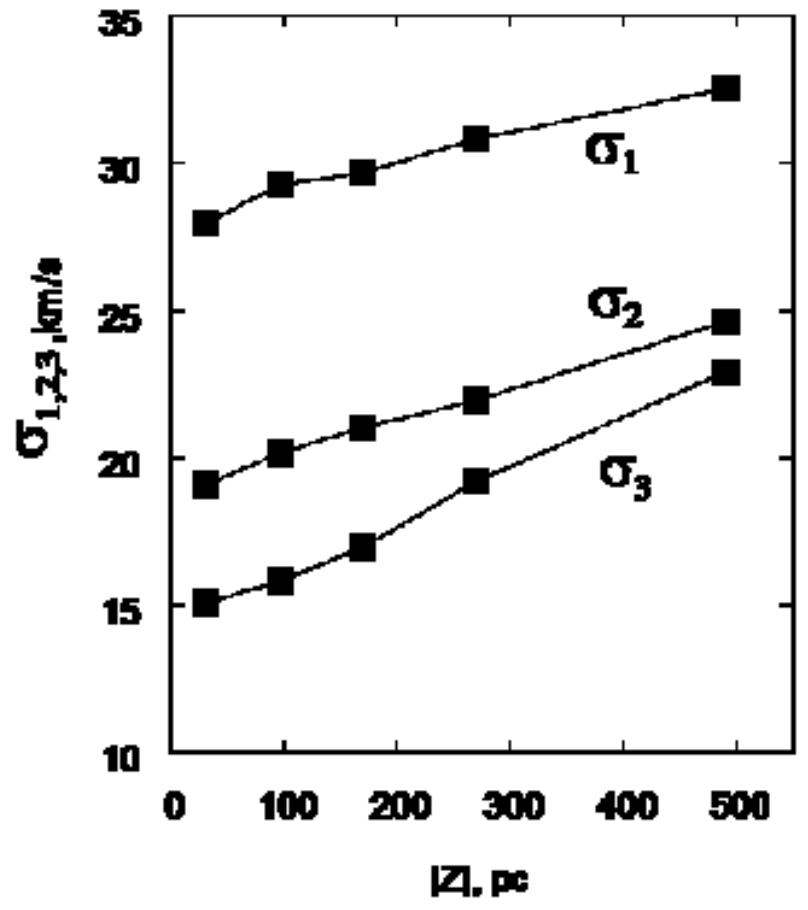

Fig. 2. Principal semiaxes of the residual velocity ellipsoid $\sigma_{1}, \sigma_{2}, \sigma_{3}$ versus $|Z|$. 

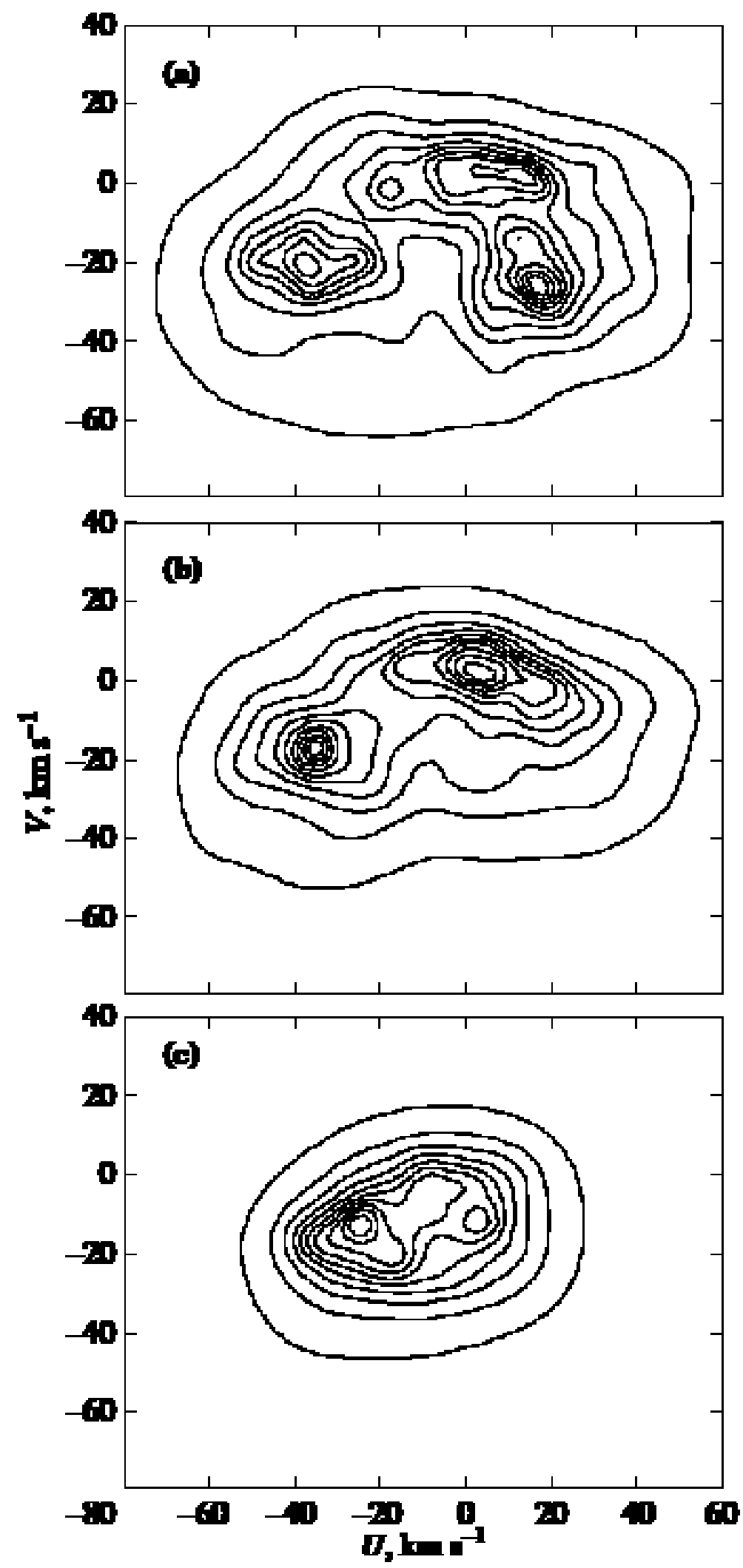

Fig. 3. Smoothed distributions of UV velocities: (a) sample (1) for $0<\Delta S$; (b) sample (2) for $-0.38<\Delta S<0$; (c) sample (3) for $-1<\Delta S<-0.38$ (see the text). 


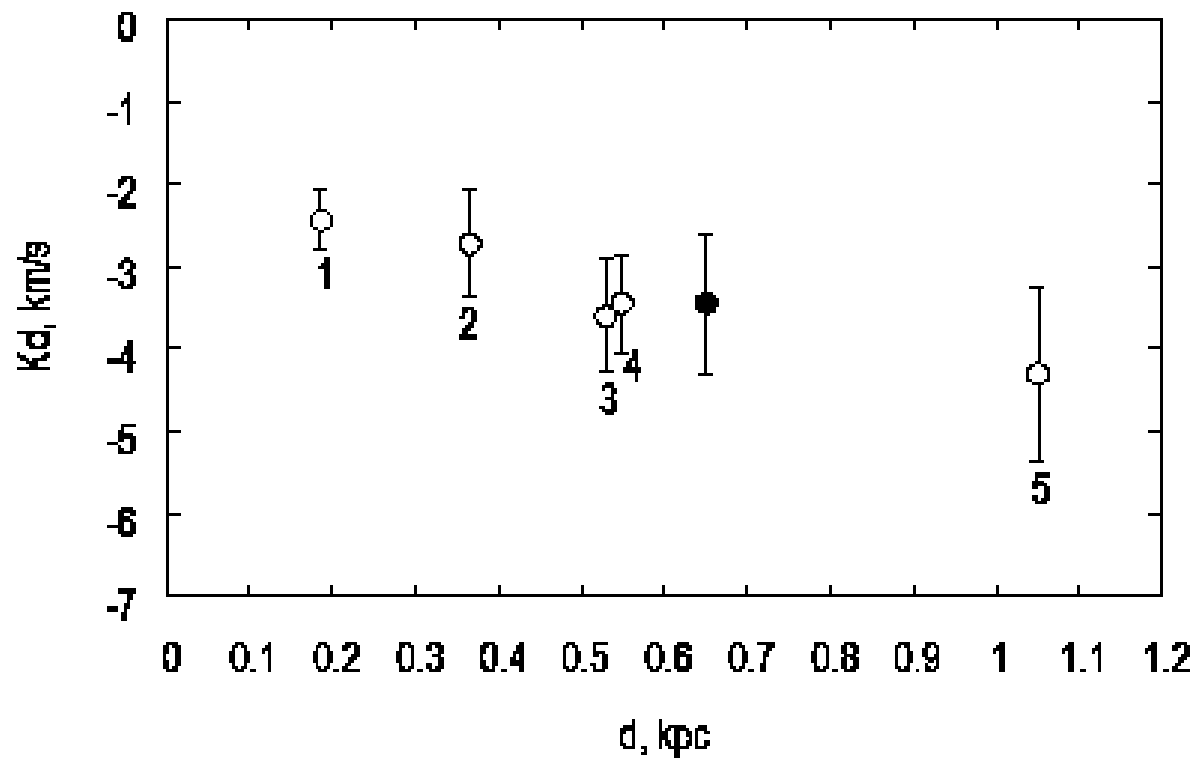

Fig. 4. Velocity $K d$ versus heliocentric distance $d$; the open circles mark the cited data (see the text) and the filled circles indicate the value of $K d$ from Table 2. 

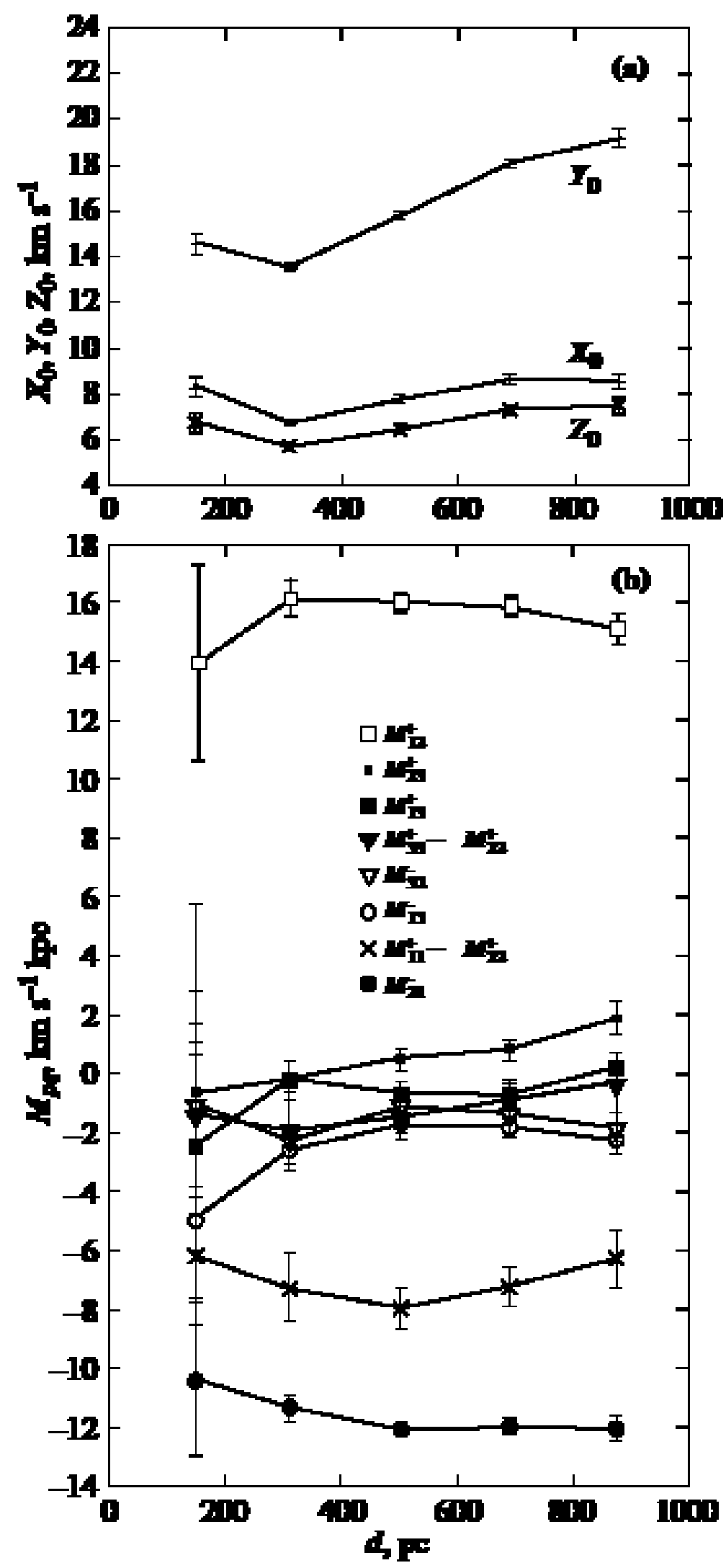

Fig. 5. Parameters of the Ogorodnikov-Milne model versus distance calculated without introducing any model errors. 

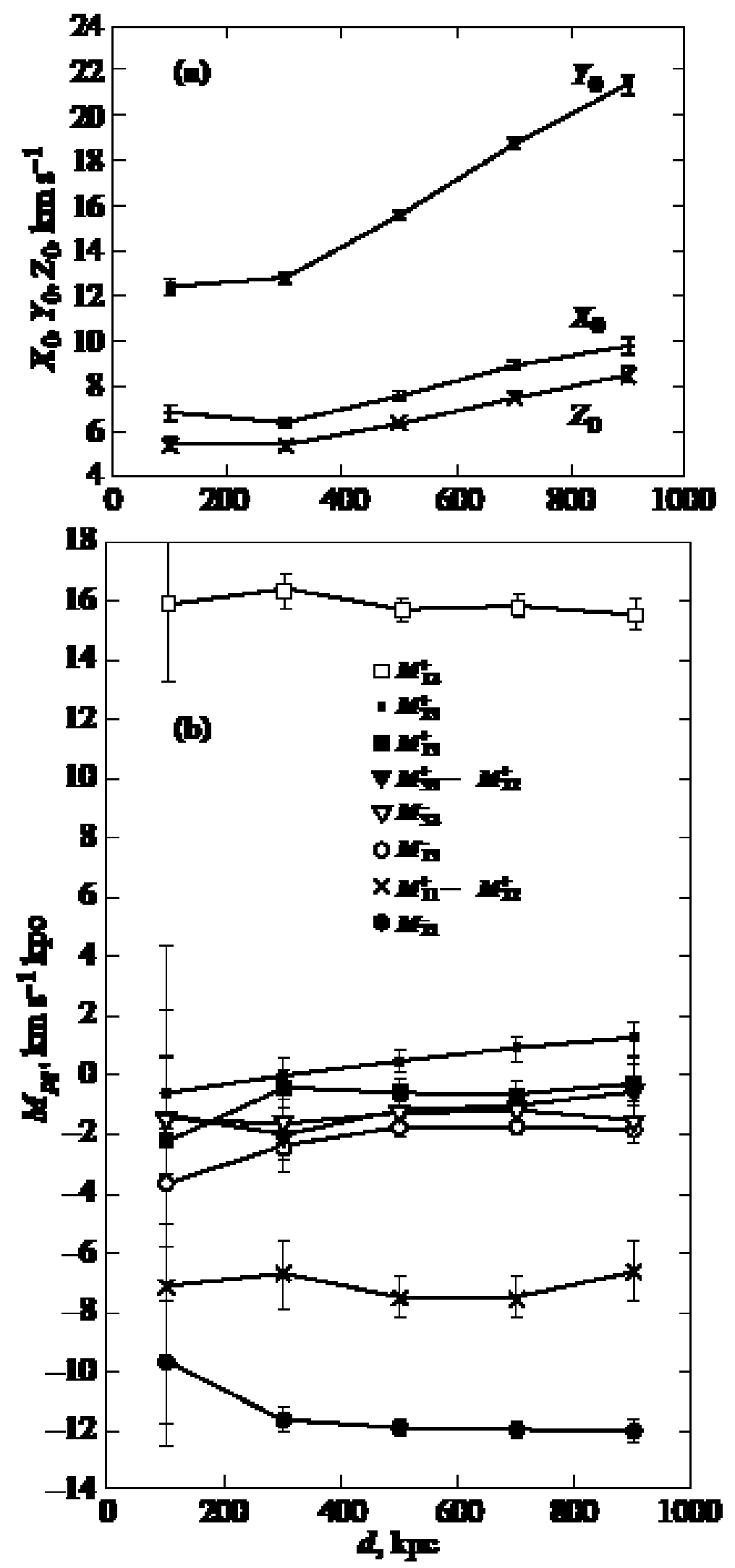

Fig. 6. Parameters of the Ogorodnikov-Milne model calculated with model distances versus distance; the random errors in the individual distance of a star are $20 \%$. 

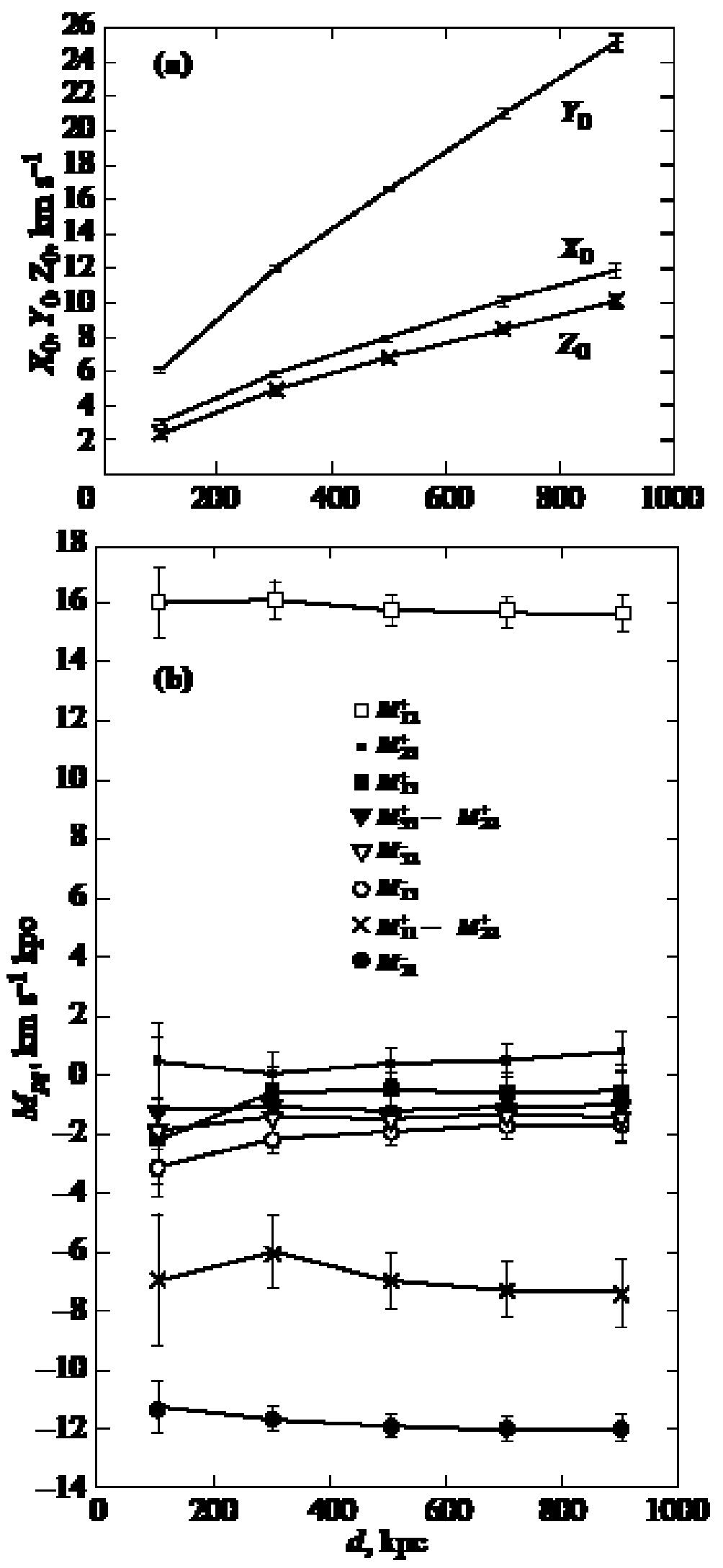

Fig. 7. Parameters of the Ogorodnikov-Milne model calculated with model distances versus distance; the random errors in the individual distance of a star are $60 \%$. 